\title{
Study on the Distribution of the Temperature Field of Flow Liquid
}

\author{
Xi Yanping ${ }^{1}$ \\ ${ }^{1}$ School of Electrical and Electric Engineering, North China Electric Power University, Beijing, \\ 102206, China
}

Keywords: ANSYS, Temperature Fields, Boundary Layer Theory

\begin{abstract}
It is quite difficult to get the temperature field of flow liquid from the three-dimensional perspective by hand calculation. But we figure out an unusual method to obtain the temperature, and we also use 3D software to simulate and test. In order to facilitate the calculation, we use cuboid bathtub as our model to study. In our model, we analyze the temperature field in an innovative perspective. We take part of the bathtub water around the faucet as a heat source based on Boundary Layer Theory, which simplifies the influence of the inlet water. We separate the temperature field into three directions and make analysis one by one to modify the difficulty of calculating it.As a result, we obtain the concrete distribution of the temperature field in three dimensions. We use the division of heat source and cooler area to get the temperature field around the faucet. Considering the similarity of the heat-dissipating pattern between bathtubs and fins, we optimize the calculation of the temperature field. Finally, we test our model with the simulation software ANSYS and the results support our theoretical calculation, which demonstrates that our model is reasonable and practical.
\end{abstract}

\section{Introduction}

It is quite difficult to get the temperature field of flow liquid from the three-dimensional perspective by hand calculation. However, if we find a suitable hand calculation method to solve the problem, it is of great significance and practical. The temperature distribution of the water is related to three ways of heat transfer: heat conduction, heat convection and radiation. We simplify different types of heat transfer to make it easier working out temperature field. Later, we have a comprehension understanding of the temperature field distribution.

\section{Assumption}

The bathtub and the bather are considered as cuboids. The bather is still in the water and water does not flow. The initial temperature is set as and distributed evenly. In the whole process, water temperature fluctuations within the scope of ups and downs $\left(38^{\circ} \mathrm{C} \sim 42^{\circ} \mathrm{C}\right)$. The temperature and humidity inside is fixed evenly and constant. Not considering the radiation heat transfer. Assuming that water is static, that is, the surface is smooth and flat, almost has no fluctuations. The bottom of the bathtub is adiabatic

\section{Models and Solutions}

At first, we assume that the bathtub is full of water and without and outflow, also nobody use the bathtub at the time. We call it Instantaneous Temperature Field. 
Considering the requirement of maintaining a constant temperature, we deem that the water temperature fluctuates within a small range. Based on general knowledge, the comfortable bathing temperature is little higher than body temperature. According to this, we set the basic water temperature as $40^{\circ} \mathrm{C}$ and the range of variation is between $38^{\circ} \mathrm{C}$ to $42^{\circ} \mathrm{C}$. The room temperature is set as $26^{\circ} \mathrm{C}$. And the initial water temperature is $t_{0}=40^{\circ} \mathrm{C}$.

According to Heat Transfer Theory, static water' s temperature field can be described byPartial Differential Equationof heat conduction:

$$
\frac{\partial t}{\partial \tau}=a\left(\frac{\partial^{2} t}{\partial x^{2}}+\frac{\partial^{2} t}{\partial y^{2}}+\frac{\partial^{2} t}{\partial z^{2}}\right)+\frac{\phi}{\rho c}
$$

Where $t$ represents the temperature of arbitrary points, $\tau$ represents the experience time of initial state, and $\alpha$ represents the heat spread speed of materials.

It is very difficult to solve partial differential equations in three dimensional space. The solution involves the homogeneous partial differential of the equations and the boundary conditions and the application of the three dimensional numerical method.

We simplify our bathtub into a cuboid with uniform thickness shell. To demonstrate better the simplification of bathtub model, we draw pictures in Figure 3.1.
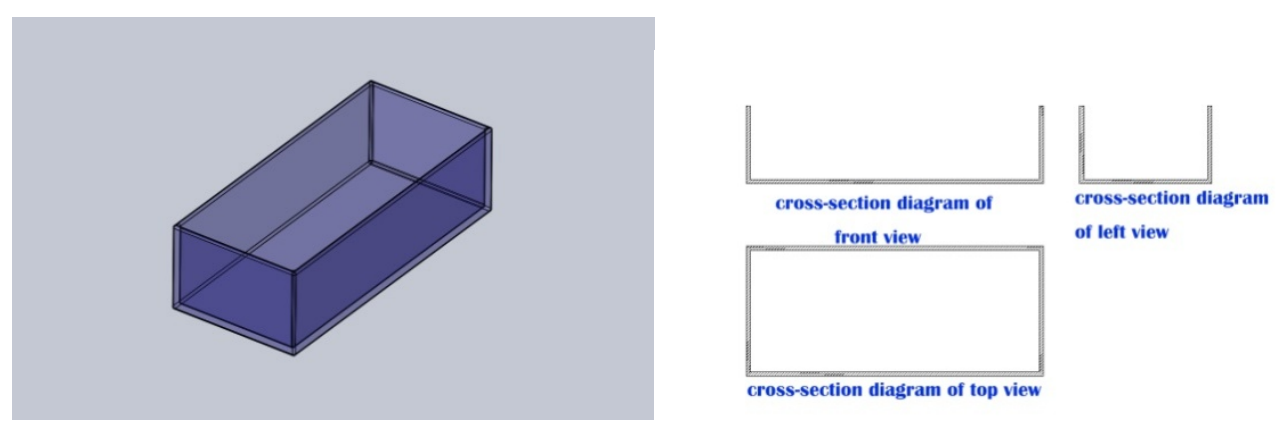

Figure 3.1

According to the size of regular bathtub, we set the size of the bathtub model, which is: $L=1.4 m, W=0.6 m, H=0.36 m$.

Heat is lost as the water flows. The father distance away from the faucet, the lower water temperature is (This will be demonstrated in the following chapters).

In order to keep the heat, we place the faucet far from the water outlet.

\section{The Division of the Temperature Field}

Put the bathtub into the rectangular coordinate system, showing in the Figure 3.2.

In Figure 3.2, $x$ axis is parallel to the longest edge of the bathtub. $y$ axis is parallel to the shortest edge of the bathtub. $z$ axis is parallel to the height of the bathtub. 


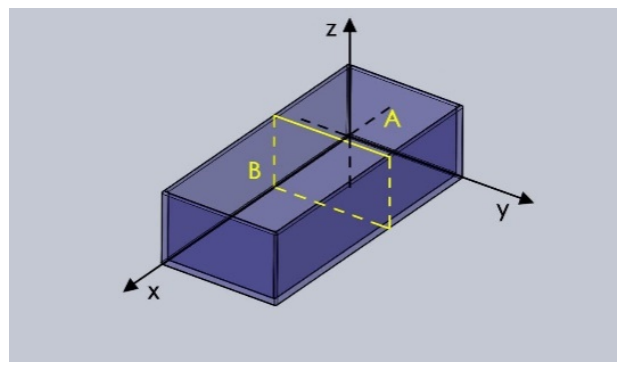

Figure3.2

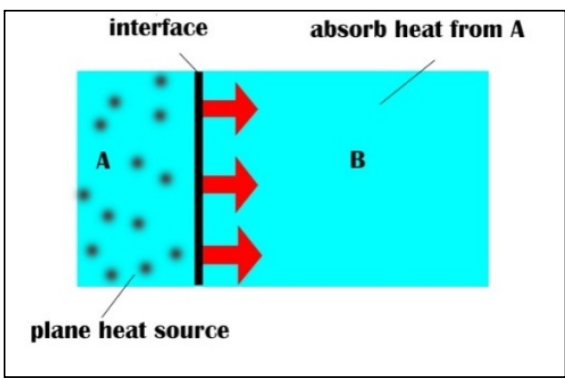

Figure3.3

Temperature field can be divided into two parts( $\left.A: 0<x<L_{0}, B: L_{0}<x<L\right)$ along $\mathrm{x}$ axis. As illustrated in Figure 4.3, section $A$ is around the faucet, while section $B$ is far away from the faucet and account for the most volume. Judging from Fluid Dynamics, section $A$ has a higher temperature. And heat spread quite quickly in section $A$ because of the interference of water flows from the faucet, Therefore, the water in section $A$ can be regarded as internal heat sources in linear distribution. As for section $B$, we ignore the effect of the faucet. However, water in section $B$ will be affected by section $A$. So we regard section $A$ as surface heat source of section $B$, that is, section $B$ absorbs heat from interface between section $A$ and section $B$. We draw the Figure 3.3 to show the specific situation.

There are mainly two heat transfer mode in the section $B$ :oneis the heat conduction of betweenwater and the wall of bathtub and the other is theheat convection between water and air. According to the assumption5, the radiation heat transfer should be ignored. The effects of these two kinds of heat transfer modes on the temperature field in section $B$ are discussed respectively.

Only considering the heat conduction between water and the wall of bathtubs.

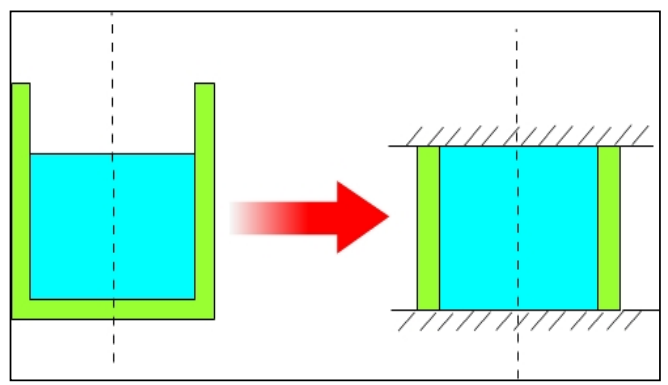

Figure 3.4

When there only exists the heat conduction betweenwater and the wall of bathtub, the upper and lower sides of the water are adiabatic, while both sides are heat conduction. The temperature 
distribution in plane zoy is symmetrical, as showed in Figure 3.4. So we only consider the change of temperature in half plane. In the middle of the plane, the temperature is horizontal distribution, which isequivalent to be adiabatic. At the middle, the temperature stays at original temperature.

In the plane showed in Figure 3.4, the distribution of temperature can be processed as one-dimensional heat conduction. Betweenwater and the wall of bathtubs, air and the wall of bathtubs, the way of heat transfer belongs to heat convection. So we have the equation as follows:

$$
q=\frac{\Delta t}{\frac{1}{h_{1}}+\frac{1}{h_{2}}+\frac{\delta}{\lambda}}
$$

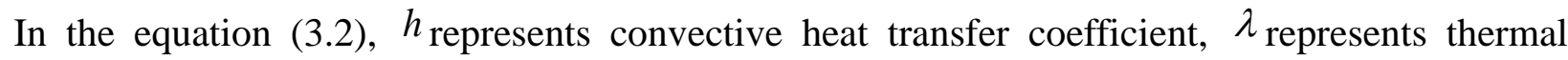
conductivity of the wall of bathtubs. $\delta$ represents the thickness of the wall of bathtubs. $\Delta t$ is the temperature difference between water and air, $q$ represents of heat flux per unit area. And we obtain the controlling equation for one dimensional heat conduction:

$$
\frac{\partial t}{\partial \tau}=a \frac{\partial^{2} t}{\partial x^{2}}
$$

Boundary conditions:

$$
\begin{aligned}
& y=0, \frac{\partial t}{\partial y}=0 \\
& y=\frac{w}{2},-\lambda \frac{\partial t}{\partial y}=q .
\end{aligned}
$$

Take the data into the equation, we can draw the Figure 3.5 of temperature distribution followed by space from the middle to the margin according to Figure 3.4.

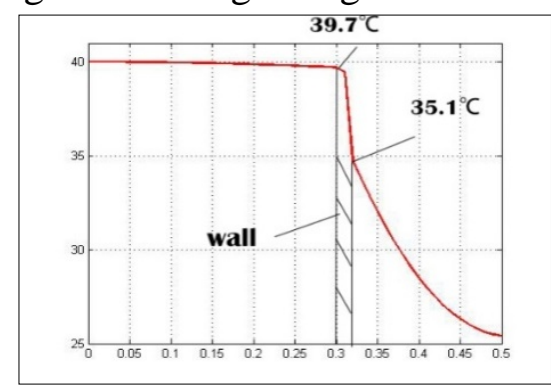

Figure 3.5

From the Figure 3.5 we can see, because the thermal conductivity of the wall is small (Defined as thermal insulation material in the national standard), the temperature decreases a lot in the wall of bathtubs. The temperature difference between the middle to the margin is $0.3^{\circ} \mathrm{C}$, which is small enough to be neglected. And the heat flux we calculate is $61.44 \mathrm{w} /\left(\mathrm{m}^{2} \cdot \mathrm{k}\right)$.

\section{The Distribution of the Mean Temperature Along the $X$ axis}

Now let's consider the distribution of the water in the bathtub along the $x$ axis.Based the discussion of 1.4, the region is distinguished by the $A-B$ interface as a surface heat source. And from the results above, there are two heat transfer modes which is convection and conduction. And 
The heat flux density of two methods vary along water flow direction. The heat transfer mode is mainly convection heat transfer, and is proportional to the difference between the liquid surface temperature and the environmental temperature.

Heat transfer in this way is very similar to fin cooling. When the fin base temperature is constant, the heat flux keeps changing along fin extension, which is proportional to the temperature difference between fin and air. So we use the equation of fin temperature field to calculate that of the bathtub.

$$
t-t_{\infty}=\left(t_{0}-t_{\infty}\right) \frac{\operatorname{ch}[m(x-H)]}{\operatorname{ch}(m H)}
$$

where $t_{0}$ and $t$ represents the temperature of point $x$ and fin base, $t_{\infty}$ represent environmental temperature, $m$ and $H$ are constant. Theequation stands for the heat dissipation performance.

Based on equation(3.4), we change the length of bathtub $H$ into the length of section $B$, while the $x$ axis is translated, so we can get the average water temperature along the $X$ axis of the distribution of the law. The equation is as follows:

$$
t-t_{\infty}=\left(t_{0}-t_{\infty}\right) \frac{\operatorname{ch}[m(x-L)]}{\operatorname{ch}\left(m\left(L-L_{0}\right)\right)}(3.5)
$$

To show the new result of our model, we use MATLAB on equation (3.4) to reveal the results in Figure 3.6.

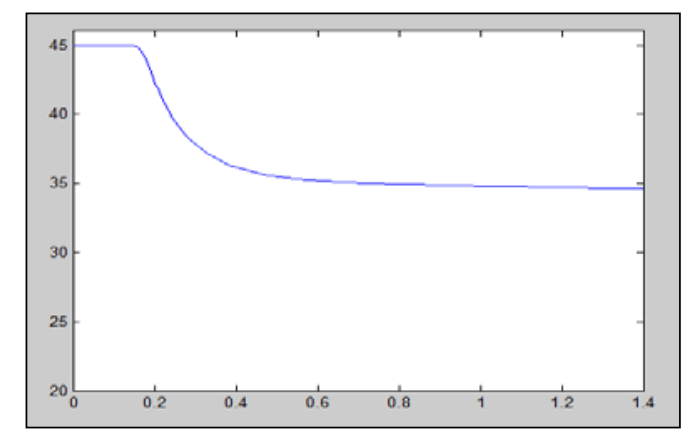

Figure 3.6

As we can seem from the Figure3.6, the temperature distribution along the $x$ axis of the bathtub begins to be more steep at first, then become more gentle subsequently. As the temperature decreases, the temperature difference between the water surface and the air gradually decreases, so as thetemperature drop rate.

The Test of Temperature Distribution Model. We test our model with the simulation software ANSYS, the results show thatthe distribution of the temperature fieldis approximately even and the error comparing with our calculation is extremely small. Along the direction of water flows, the temperature gradually decreased. The error of the outlet temperature between the simulation and our calculation is $0.6^{\circ} \mathrm{C}$, indicating that the results support our theoretical calculation.It demonstrates that our model is reasonable and practical.

\section{Conclusion}

We develop a model based on three dimensional view. The water temperature is mainly distributed on the high axis. While on the each horizontal plane, the temperature field distribute evenly. The 
error between our calculation and simulation is $0.6^{\circ} \mathrm{C}$, which proves our conclusion is consistent with the reality.

\section{Reference}

[1] Yunnus A. Cengel. Hear Transfer[M]. Second Edition, New York: Mc Graw-Hill Company, 2003.

[2] Pelzer H. Ingeniervermessung[M]. Stuttgart: Konrad Wittwer,2001.

[3 ] Shimin Mao. Discussion on the formula and application of heat dissipation of water.[J]. Journal of Hydraulic Engineering, 2004.

[4] Congzhi Zhou, Steven C.Chapra. Simulation of thermal structure and evaporation of Lake[J]. Chinese Journal of Environmental Science, 1994, 15:33 37. 